\title{
SYNTHESIS AND BIOACTIVITY OF ROTENONE OXIME $O$-ETHER DERIVATIVES
}

\author{
Gao Cao*, Zhen Zhou and Ying Wang \\ School of Chemistry and Chemical Engineering, Guangdong Pharmaceutical University, \\ Guangzhou 510006, P. R. China
}

(Received July 20, 2011; revised March 12, 2012)

\begin{abstract}
A series of rotenone oxime $O$-ether derivatives were synthesized and characterized. All compounds were tested for their insecticidal, miticidal and fungicidal activities against the selected pests and compared with those of rotenone. The results of biological tests show that the rotenone oxime $O$-ether derivatives have improved insecticidal but weak miticidal activities against $M$. eparate, $N$. cincticeps, $T$. urticae compared with rotenone. The disustituted oxime $O$-ethers showed novel fungicidal activity against $R$. solani with an inhibition of $80.7-95.2 \%$.
\end{abstract}

KEY WORDS: Rotenone oxime $O$-ether, Synthesis, Insecticidal activity, Miticidal activity, Fungicidal activity

\section{INTRODUCTION}

Pesticides play an important role in our lives, not only for crop protection in agriculture, but also for human health. However, the unrestricted use of highly toxic pesticides over several decades has resulted in the accumulation of pesticides in the soil and their dispersion into the environment [1]. Rotenone (Figure 1) is an abundant natural product occurring in the roots of tropical plants belonging to the Leguminosae family [2]. It has been widely used as an insecticide, acaricide and piscicide because of its broad spectrum of toxicity, and hence has been a valuable alternative to synthetic pesticides [3, 4]. However, rotenone has a serious limitation to its widespread usage due to its susceptibility towards ultraviolet rays or solar irradiation. Owing to rotenone's high photolability, either breaking down or isomerizing in the presence of sunlight will decrease its bioactivities under field conditions, resulting in poor persistence in the environment and inadequate field performance [5].

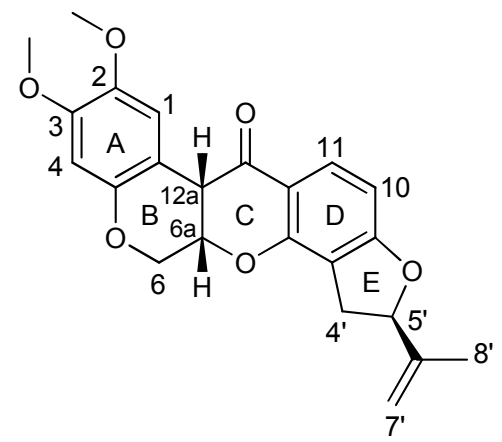

Figure 1. Structure of rotenone with the atoms labeling scheme used in the spectral elucidation.

Imitating and modifying the chemistry of biologically active natural products is an alternative approach for developing more efficient and less toxic pesticides. Several investigations of structure-activity relationships have been reported, using mainly natural

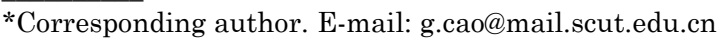


rotenoids and their derivatives [6-9]. The high inhibitory potency of rotenone is associated with the compounds containing the A-B-C-D-E five rings system with the cis-6a $\beta, 12 \mathrm{a} \beta-\mathrm{B} / \mathrm{C}$ ring juncture, this arrangement result in an approximately $V$-shaped molecule with a certain angle; Another important structural feature is that 2,3-dimethoxy substitution on the ring $\mathrm{A}$ and a suitable substituent on the ring $\mathrm{E}[10,11]$. Modifications at the $\mathrm{B}$ and $\mathrm{C}$ rings of rotenone are permissible as long as they do not seriously affect the preferred configuration of the $\mathrm{B} / \mathrm{C}$ ring juncture. In order to improve the biological activities and photostabilization of rotenone, we designed and synthesized a series of rotenone oxime $O$-ether derivatives using natural rotenone as a substrate. Furthermore, all the compounds prepared, along with the parent compound rotenone, were tested for their insecticidal, miticidal and fungicidal activities against selected pathogens.

\section{RESULTS AND DISCUSSION}

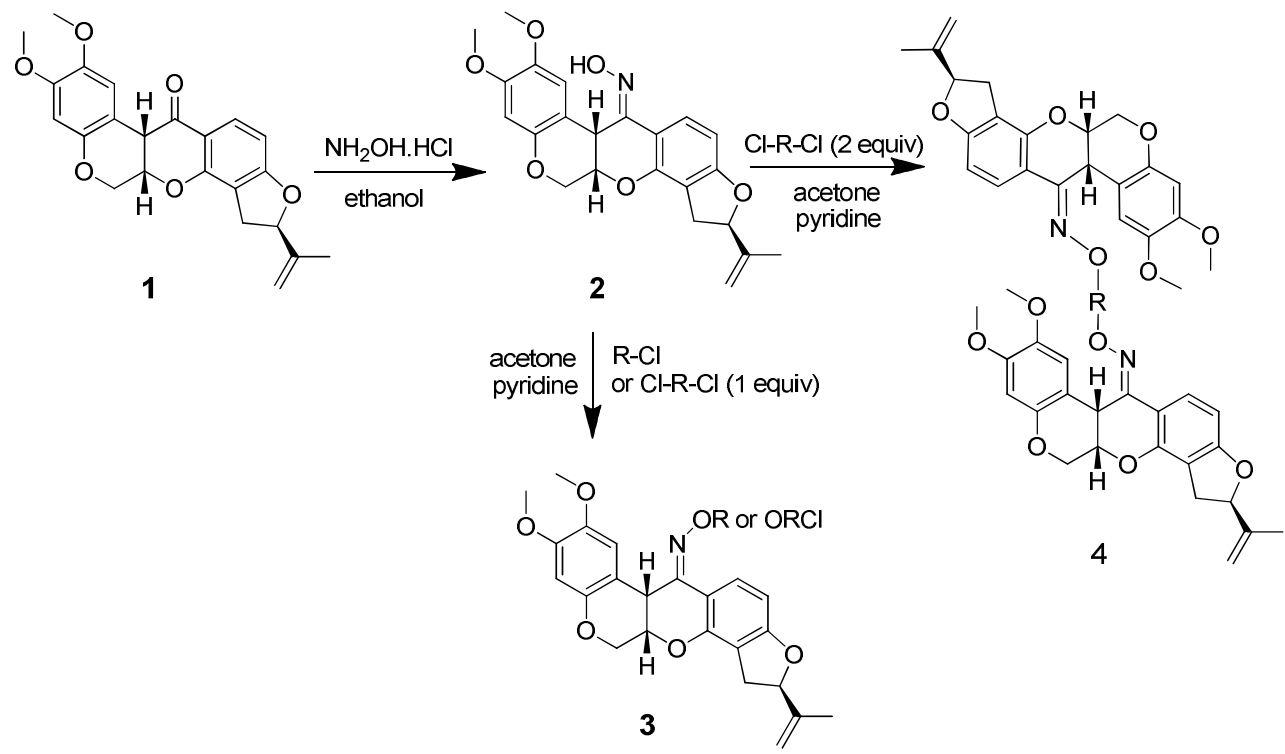<smiles>[R18]=C1C=CC(C)=CC1</smiles><smiles>[R19]=C=CC1=CC=C(C)CC1</smiles><smiles></smiles>

3d: $R=$<smiles>CCc1ncc(Cl)s1</smiles><smiles></smiles><smiles>[B]=C=[GeH]</smiles><smiles>Cc1cc(C)c(C[C]=[Ru][Ba])cc1C</smiles><smiles>[R]Cc1ccc(C)cc1</smiles><smiles>[R16]=CCc1ccc(-c2ccc(C)cc2)cc1</smiles><smiles>[C]C#[R]Cc1cc(C)c(C)cc1C</smiles>

Scheme 1. Synthetic pathway to rotenone oxime $O$-ether derivatives (3a-3g and $\mathbf{4 a - 4 c )}$. 
Compound 2 was prepared by the reaction of rotenone and hydroxylamine in ethanol (Scheme 1) by the procedure reported previously, and its melting point is in agreement with the literature value $\left(237-239{ }^{\circ} \mathrm{C}\right)$ [12]. Furthermore, X-ray diffraction results show that compound 2 exists in the form of $E$-isomer with the isopropenyl group in an equatorial position [13]. In the process of preparing $\mathbf{3}$, the phase transfer catalyst plays an important role in improving the yields and shortening the reaction time. Compounds $\mathbf{3 a - 3 g}$ were prepared in an alkaline medium, and the addition of potassium iodide accelerated the reactions via the halogenexchange. Table 1 summarized the time of reactions, yield, and melting point of $\mathbf{3 a}-\mathbf{3 g}, \mathbf{4 a}-\mathbf{4} \mathbf{c}$ derivatives.

Table 2 shows the insecticidal and miticidal activities of rotenone and the oxime $O$-ethers against the selected insects Mythimna separata (M. separate), Nephotettix cincticeps ( $N$. cincticeps), Aphis fabae (A. fabae) and mite Tetranychus urticae (T. urticae). Generally, most of the rotenone oxime $O$-ether derivatives exhibited insecticidal and miticidal activities against $M$. eparate, $N$. cincticeps and T. urticae, comparable to the parent compound rotenone. It is notable that all compounds, including rotenone, have no insecticidal activity against A. fabae. Of the test compounds, compounds $\mathbf{4 a}$ and $\mathbf{4} \mathbf{c}$ are the most active.

Table 1. Reaction time, yield and m.p. data in synthesis of rotenone oxime $O$-ethers.

\begin{tabular}{|c|c|c|c|}
\hline Product & $\begin{array}{c}\text { Reaction } \\
\text { time }(\mathrm{h})\end{array}$ & Yield $(\%)$ & M.p. $\left({ }^{\circ} \mathrm{C}\right)$ \\
\hline 3a & 36 & 73.0 & $226-228$ \\
\hline 3b & 10 & 68.8 & $178-180$ \\
\hline 3c & 20 & 75.1 & $191-193$ \\
\hline $\mathbf{3 d}$ & 10 & 71.9 & $188-190$ \\
\hline $\mathbf{3 e}$ & 12 & 41.0 & $>260$ \\
\hline $\mathbf{3 f}$ & 12 & 57.3 & $165-167$ \\
\hline $\mathbf{3 g}$ & 13 & 60.0 & $190-192$ \\
\hline $\mathbf{4 a}$ & 23 & 55.6 & $>260$ \\
\hline $\mathbf{4 b}$ & 25 & 44.5 & $>260$ \\
\hline $\mathbf{4 c}$ & 25 & 54.2 & $>260$ \\
\hline
\end{tabular}

Table 2. Insecticidal and miticidal activities of rotenone oxime $O$-ether derivatives.

\begin{tabular}{|c|c|c|c|c|}
\hline \multirow{2}{*}{ Compound } & \multicolumn{4}{|c|}{ Mortality (\%) } \\
\cline { 2 - 5 } & M. separata & N. cincticeps & T. urticae & A. fabae \\
\hline Rotenone & 43.2 & 21.5 & 20.3 & 0 \\
\hline 3a & 35.2 & 25.4 & 13.3 & 0 \\
\hline 3b & 40.8 & 30.5 & 14.2 & 0 \\
\hline 3c & 34.6 & 23.5 & 12.9 & 0 \\
\hline 3d & 5.0 & 4.8 & 32.6 & 0 \\
\hline 3e & 31.5 & 18.4 & 16.7 & 0 \\
\hline 3f & 30.4 & 20.5 & 15.6 & 0 \\
\hline 3g & 45.3 & 19.0 & 15.2 & 0 \\
\hline 4a & 46.7 & 22.7 & 27.1 & 0 \\
\hline 4b & 42.1 & 21.4 & 20.8 & 0 \\
\hline 4c & 43.5 & 22.1 & 30.2 & 0 \\
\hline
\end{tabular}

For the structure-activity relationship analysis, compounds $\mathbf{3 a - 3 g}$ and $\mathbf{4 a - 4} \mathbf{c}$ were defined as monosubstituted derivatives and disubstituted derivatives respectively. Comparing the bioactivities of the monosubstituted derivatives (3a-3g), it was found that suitable substituents 
on the phenyl group are so important that a huge difference has been observed. For example, $\mathbf{3 e}$ and 3f have moderate antifungal activity against Rhizoctonia solani ( $R$. solani), but 3g lost antifungal activity completely (Table 3 ). The oxime $O$-ethers, in which $\mathrm{R}$ is a disubstituted group, showed a higher activity than those in which $\mathrm{R}$ is a monosubstituted group. For example, $\mathbf{4 a}, \mathbf{4 b}$ and $\mathbf{4 c}$ have particularly excellent fungicidal activity against $R$. solani, with an inhibition rate of $80.7-95.2 \%$, while the monosubstituted oxime ethers $\mathbf{3 a}, \mathbf{3 c}, \mathbf{3 g}$ and rotenone have nearly no inhibition effect on any fungicidal activity. This information can be useful for designing new macrocyclic fungicides. From the different inhibition effects of $\mathbf{4 a}, \mathbf{4 b}$ and $\mathbf{4 c}$, the fungicidal activity of disubstituted oxime ethers is also closely associated with $\mathrm{R}$ on the bridged chain. In addition, the results show that the introduction of the thiazole ring (3d) provides little help but is actually harmful in terms of improving the bioactivity. Further studies on the biological activity and structure-activity relationships of this series of compounds are in progress.

Table 3. Fungicidal activity of the test compounds against the selected pathogen species ( $500 \mathrm{mg} / \mathrm{L}$ ).

\begin{tabular}{|c|c|c|c|c|c|c|c|}
\hline \multirow{2}{*}{ Compound } & \multicolumn{7}{|c|}{ Inhibition (\%) } \\
\cline { 2 - 9 } & G. zeae & P. capsici & P. oryzae & B. cinerea & R. solani & S. sclerotiorum & E. graminis \\
\hline Rotenone & 0 & 0 & 2.9 & 0 & 0 & 0 & 10.0 \\
\hline 3a & 0 & 0 & 0 & 0 & 0 & 0 & 0 \\
\hline 3b & 6.8 & 55.1 & 25.4 & 0 & 40.1 & 0 & 10.0 \\
\hline 3c & 0 & 39.4 & 5.6 & 0 & 0 & 0 & 0 \\
\hline 3d & 0 & 0 & 7.1 & 0 & 8.5 & 0 & 0 \\
\hline 3e & 3.4 & 0 & 0 & 0 & 60.2 & 7.5 & 0 \\
\hline 3f & 6.4 & 0 & 0 & 0 & 60.5 & 5.6 & 0 \\
\hline 3g & 2.6 & 0 & 3.1 & 0 & 0 & 1.2 & 0 \\
\hline 4a & 0 & 7.8 & 6.0 & 9.1 & 90.6 & 9.3 & 0 \\
\hline 4b & 0 & 0 & 0 & 6.4 & 95.2 & 3.7 & 0 \\
\hline 4c & 0 & 3.5 & 6.2 & 3.1 & 80.7 & 5.4 & 0 \\
\hline
\end{tabular}

\section{CONCLUSIONS}

The molecular design, synthesis, insecticidal, miticidal and fungicidal activities of a series of rotenone oxime $O$-ether derivatives have been demonstrated. Some compounds were found to display higher insecticidal and miticidal activities against $M$. separata, $N$. cincticeps and $T$. urticae than rotenone. Derivatives $\mathbf{4 a}, \mathbf{4 b}$ and $\mathbf{4 c}$ not only showed general insecticidal activity but also have a novel fungicidal activity, especial against $R$. solani at a dosage of $500 \mathrm{mg} / \mathrm{L}$. These derivatives were identified as being the most promising candidates for further study. Further structural optimization and fungicidal activities studies on these rotenoid analogues are ongoing in our laboratories.

\section{General}

\section{EXPERIMENTAL}

Rotenone (purity $=95-98 \%$ ) was purchased from Sigma Aldrich (Steinheim, Germany). Other reagents were analytical grade. Melting points were determined using a RY-1 melting point apparatus and were given uncorrected. Infrared spectra were recorded on an Avatar-360FT infrared spectrometer using potassium bromide discs. ${ }^{1} \mathrm{H}$ NMR spectra were recorded on a Bruker Avance-400 superconductor nuclear magnetic resonance instrument, with chemical shift $(\delta)$ values reported in ppm relative to tetramethylsilane (TMS) as an internal standard. Elemental analysis was performed with an Elemental Vario EL apparatus. 
Synthetic procedures for compound $3 a-3 g, 4 a-4 c$

A solution of chloroalkane ( $\mathrm{RCl}$ or $\left.\mathrm{RCl}_{2}, 0.01 \mathrm{~mol}\right)$ in anhydrous pyridine $(5 \mathrm{~mL})$ was added dropwise to a stirred solution of compound $2(0.01 \mathrm{~mol}, 0.02 \mathrm{~mol}$ for $\mathbf{4 a}-\mathbf{4 c})$ in acetone $(20 \mathrm{~mL})$ at room temperature $\left(20^{\circ} \mathrm{C}\right)$. Then the mixture was stirred and heated to reflux. All reactions were performed under an atmosphere of nitrogen. After the reaction finished, the reaction mixture was concentrated. The residual solution was purified by column chromatography $(2 \times 22$ inch silica column) using petroleum ether and ethyl acetate ( $3: 1$ by volume) as eluent. The main peak was concentrated to afford $\mathbf{3 a - 3 g}, \mathbf{4 a - 4 c}$.

\section{Characterization data of the rotenone oxime O-ether derivatives}

Compound 3a. Orange solid. ${ }^{1} \mathrm{H}$ NMR $\left(\mathrm{CDCl}_{3}, 400 \mathrm{MHz}\right) \delta: 1.75\left(\mathrm{~s}, 3 \mathrm{H}, 8^{\prime}-\mathrm{CH}_{3}\right), 2.91(\mathrm{dd}, J=$ $\left.8.0 \mathrm{~Hz}, J=16.0 \mathrm{~Hz}, 1 \mathrm{H}, 4^{\prime}-\mathrm{H}\right), 3.27$ (dd, $\left.J=10.0 \mathrm{~Hz}, J=16.0 \mathrm{~Hz}, 1 \mathrm{H}, 4^{\prime}-\mathrm{H}\right), 3.50$ (s, 3H, $\left.\mathrm{OCH}_{3}\right), 3.78\left(\mathrm{~s}, 3 \mathrm{H}, \mathrm{OCH}_{3}\right), 4.21(\mathrm{~d}, J=12.0 \mathrm{~Hz}, 1 \mathrm{H}, 6-\mathrm{H}), 4.48(\mathrm{t}, J=2.8 \mathrm{~Hz}, 1 \mathrm{H}, 12 \mathrm{a}-\mathrm{H})$, $4.55(\mathrm{dd}, J=12.0 \mathrm{~Hz}, J=2.4 \mathrm{~Hz}, 1 \mathrm{H}, 6-\mathrm{H}), 4.80$ (d, $J=2.4 \mathrm{~Hz}, 1 \mathrm{H}, 6 \mathrm{a}-\mathrm{H}), 4.90$ (s, 1H, 7'-H), $5.06\left(\mathrm{~s}, 1 \mathrm{H}, 7^{\prime}-\mathrm{H}\right), 5.14\left(\mathrm{t}, J=8.8 \mathrm{~Hz}, 1 \mathrm{H}, 5^{\prime}-\mathrm{H}\right), 5.28(\mathrm{dd}, J=12.0 \mathrm{~Hz}, J=12.0 \mathrm{~Hz}, 2 \mathrm{H}$, $\left.\mathrm{NOCH}_{2}\right), 6.39(\mathrm{~s}, 1 \mathrm{H}, 4-\mathrm{H}), 6.44(\mathrm{~d}, J=8.4 \mathrm{~Hz}, 1 \mathrm{H}, 10-\mathrm{H}), 6.77(\mathrm{~s}, 1 \mathrm{H}, 1-\mathrm{H}), 7.38(\mathrm{~m}, 3 \mathrm{H}$, $\left.\mathrm{C}_{6} \mathrm{H}_{5} 3,4,5-\mathrm{H}\right), 7.47\left(\mathrm{~d}, J=6.4 \mathrm{~Hz}, 2 \mathrm{H}, \mathrm{C}_{6} \mathrm{H}_{5} 2,6-\mathrm{H}\right), 7.79(\mathrm{~d}, J=8.4 \mathrm{~Hz}, 1 \mathrm{H}, 11-\mathrm{H})$. Anal. calcd for $\mathrm{C}_{30} \mathrm{H}_{29} \mathrm{NO}_{6}$ : C, $72.13 \%, \mathrm{H}, 5.85 \%, \mathrm{~N}, 2.80 \%$. Found: C, $71.80 \%, \mathrm{H}, 5.49 \%, \mathrm{~N}, 2.80 \%$. IR (KBr) $v / \mathrm{cm}^{-1}: 3062,2929,2982,2852,1620,1509,1198,741$.

Compound $3 \boldsymbol{b}$. White solid. ${ }^{1} \mathrm{H}$ NMR $\left(\mathrm{CDCl}_{3}, 400 \mathrm{MHz}\right) \delta: 1.75\left(\mathrm{~s}, 3 \mathrm{H}, 8^{\prime}-\mathrm{CH}_{3}\right), 2.91$ (dd, $J=$ $\left.8.0 \mathrm{~Hz}, J=16.0 \mathrm{~Hz}, 1 \mathrm{H}, 4^{\prime}-\mathrm{H}\right), 3.27$ (dd, $\left.J=9.6 \mathrm{~Hz}, J=16.0 \mathrm{~Hz}, 1 \mathrm{H}, 4^{\prime}-\mathrm{H}\right), 3.50$ (s, 3H, $\left.\mathrm{OCH}_{3}\right), 3.78\left(\mathrm{~s}, 3 \mathrm{H}, \mathrm{OCH}_{3}\right), 4.21(\mathrm{~d}, J=12.0 \mathrm{~Hz}, 1 \mathrm{H}, 6-\mathrm{H}), 4.49(\mathrm{~d}, J=2.4 \mathrm{~Hz}, 1 \mathrm{H}, 12 \mathrm{a}-\mathrm{H})$, 4.57 (dd, $J=12.0 \mathrm{~Hz}, J=2.4 \mathrm{~Hz}, 1 \mathrm{H}, 6-\mathrm{H}), 4.79$ (d, $J=3.2 \mathrm{~Hz}, 1 \mathrm{H}, 6 \mathrm{a}-\mathrm{H}), 4.90$ (s, 1H, 7'-H), $5.06\left(\mathrm{~s}, 1 \mathrm{H}, 7^{\prime}-\mathrm{H}\right), 5.15\left(\mathrm{t}, J=9.2 \mathrm{~Hz}, 1 \mathrm{H}, 5^{\prime}-\mathrm{H}\right), 5.20 \sim 5.33\left(\mathrm{~m}, 3 \mathrm{H},=\mathrm{CH}_{2}, \mathrm{OCH}_{2}\right), 5.75(\mathrm{~d}, J$ $\left.=17.6 \mathrm{~Hz}, 1 \mathrm{H},=\mathrm{CH}_{2}\right), 6.39(\mathrm{~s}, 1 \mathrm{H}, 4-\mathrm{H}), 6.45(\mathrm{~m}, 2 \mathrm{H}, 1-\mathrm{H}, 10-\mathrm{H}), 6.71(\mathrm{dd}, J=10.8 \mathrm{~Hz}, J=$ $21.6 \mathrm{~Hz},=\mathrm{CH}), 7.42\left(\mathrm{q}, J=8.0 \mathrm{~Hz}, 4 \mathrm{H}, \mathrm{C}_{6} \mathrm{H}_{4}\right), 7.79(\mathrm{~d}, J=8.8 \mathrm{~Hz}, 1 \mathrm{H}, 11-\mathrm{H})$. Anal. calcd for $\mathrm{C}_{32} \mathrm{H}_{31} \mathrm{NO}_{6}: \mathrm{C}, 73.13 \%, \mathrm{H}, 5.94 \%, \mathrm{~N}, 2.66 \%$. Found: C, 73.65\%, H, 6.13\%, N, 2.58\%. IR (KBr) $v / \mathrm{cm}^{-1}: 3081,3063,2976,2935,2877,2853,1620,1511,1198,900,742$.

Compound $3 \boldsymbol{c}$. White solid. ${ }^{1} \mathrm{H}$ NMR $\left(\mathrm{CDCl}_{3}, 400 \mathrm{MHz}\right) \delta: 1.75\left(\mathrm{~s}, 3 \mathrm{H}, 8^{\prime}-\mathrm{CH}_{3}\right), 2.91$ (dd, $J=$ $\left.8.0 \mathrm{~Hz}, J=16.0 \mathrm{~Hz}, 1 \mathrm{H}, 4^{\prime}-\mathrm{H}\right), 3.27$ (dd, $\left.J=10.0 \mathrm{~Hz}, J=16.0 \mathrm{~Hz}, 1 \mathrm{H}, 4^{\prime}-\mathrm{H}\right), 3.58$ (s, 3H, $\left.\mathrm{OCH}_{3}\right), 3.79\left(\mathrm{~s}, 3 \mathrm{H}, \mathrm{OCH}_{3}\right), 4.23(\mathrm{~d}, J=12.0 \mathrm{~Hz}, 1 \mathrm{H}, 6-\mathrm{H}), 4.49(\mathrm{~s}, 1 \mathrm{H}, 12 \mathrm{a}-\mathrm{H}), 4.58(\mathrm{~d}, J=$ $12.0 \mathrm{~Hz}, 1 \mathrm{H}, 6 \mathrm{H}), 4.74(\mathrm{~s}, 1 \mathrm{H}, 6 \mathrm{a}-\mathrm{H}), 4.90$ (s, 1H, 7'-H), 5.05 (s, 1H, 7'-H), 5.15 (t, J = 8.8 Hz, $\left.1 \mathrm{H}, 5^{\prime}-\mathrm{H}\right), 5.26\left(\mathrm{~s}, 2 \mathrm{H}, \mathrm{OCH}_{2}\right), 6.39(\mathrm{~s}, 1 \mathrm{H}, 4-\mathrm{H}), 6.41(\mathrm{~s}, 1 \mathrm{H}, 1-\mathrm{H}), 6.44(\mathrm{~d}, J=8.8 \mathrm{~Hz}, 1 \mathrm{H}, 10-$ $\mathrm{H}), 7.37\left(\mathrm{~d}, J=8.0 \mathrm{~Hz}, 1 \mathrm{H}, \mathrm{C}_{5} \mathrm{H}_{3} \mathrm{~N} 5-\mathrm{H}\right), 7.72(\mathrm{~d}, J=8.8 \mathrm{~Hz}, 1 \mathrm{H}, 11-\mathrm{H}), 7.82(\mathrm{~d}, J=8.0 \mathrm{~Hz}$, $1 \mathrm{H}, \mathrm{C}_{5} \mathrm{H}_{3} \mathrm{~N}$ 4-H), 8.52 (s, $1 \mathrm{H}, \mathrm{C}_{5} \mathrm{H}_{3} \mathrm{~N}$ 2-H). Anal. calcd for $\mathrm{C}_{29} \mathrm{H}_{27} \mathrm{~N}_{2} \mathrm{ClO}_{6}: \mathrm{C}, 65.11 \%, \mathrm{H}$, $5.09 \%$, N, 5.24\%. Found: C, 64.92\%, H, 5.21\%, N, 5.28\%. IR (KBr) v/cm ${ }^{-1}: 3063,2963,2930$, 2879, 2856, 1780, 1621, 1514, 1199, 739 .

Compound 3d. White solid. ${ }^{1} \mathrm{H}$ NMR $\left(\mathrm{CDCl}_{3}, 400 \mathrm{MHz}\right) \delta: 1.76\left(\mathrm{~s}, 3 \mathrm{H}, \mathrm{CH}_{3}\right), 2.89-2.95(\mathrm{~m}$, $\left.1 \mathrm{H}, 4^{\prime}-\mathrm{CH}_{2}\right), 3.25-3.32\left(\mathrm{~m}, 1 \mathrm{H}, 4^{\prime}-\mathrm{CH}_{2}\right), 3.47\left(\mathrm{~s}, 3 \mathrm{H}, \mathrm{OCH}_{3}\right), 3.78\left(\mathrm{~s}, 3 \mathrm{H}, \mathrm{OCH}_{3}\right), 4.22(\mathrm{~d}, J=$ $12.0 \mathrm{~Hz}, 1 \mathrm{H}, 6-\mathrm{H}), 4.51$ (bs, $1 \mathrm{H}, 12 \mathrm{a}-\mathrm{H}), 4.59(\mathrm{dd}, J=3.0 \mathrm{~Hz}, J=12.0 \mathrm{~Hz}, 1 \mathrm{H}, 6-\mathrm{H}), 4.72(\mathrm{~d}, J$ $=3.0 \mathrm{~Hz}, 1 \mathrm{H}, 6 \mathrm{a}-\mathrm{H}), 4.91\left(\mathrm{~s}, 1 \mathrm{H}, 7^{\prime}-\mathrm{H}\right), 5.06\left(\mathrm{~s}, 1 \mathrm{H}, 7^{\prime}-\mathrm{H}\right), 5.15\left(\mathrm{t}, 1 \mathrm{H}, J=8.4 \mathrm{~Hz}, 5^{\prime}-\mathrm{H}\right), 5.33$ $\left(\mathrm{s}, 2 \mathrm{H}, \mathrm{OCH}_{2}\right), 6.31(\mathrm{~s}, 1 \mathrm{H}$, thiazole ring $\mathrm{H}), 6.40(\mathrm{~s}, 1 \mathrm{H}, 4-\mathrm{H}), 6.50(\mathrm{~d}, J=8.4 \mathrm{~Hz}, 1 \mathrm{H}, 10-\mathrm{H})$, $7.56(\mathrm{~s}, 1 \mathrm{H}, 1-\mathrm{H}), 7.80(\mathrm{~d}, J=8.4 \mathrm{~Hz}, 1 \mathrm{H}, 11-\mathrm{H})$. Anal. calcd for $\mathrm{C}_{28} \mathrm{H}_{27} \mathrm{ClN}_{2} \mathrm{O}_{6} \mathrm{~S}: \mathrm{C}, 60.59 \%$, $\mathrm{H}, 4.90 \%$, N, 5.05\%. Found: C, 60.72\%, H, 5.02\%, N, 4.96\%. IR (KBr) v/cm $\mathrm{cm}^{-1}: 3016,2971$, 2936, 2876, 2850, 1617, 1485, 1201, 982, 738 . 
Compound 3e. White solid. ${ }^{1} \mathrm{H}$ NMR $\left(\mathrm{CDCl}_{3}, 400 \mathrm{MHz}\right) \delta: 1.75\left(\mathrm{~s}, 3 \mathrm{H}, 8{ }^{\prime}-\mathrm{CH}_{3}\right), 2.92$ (dd, $J=$ $\left.8.0 \mathrm{~Hz}, J=16.0 \mathrm{~Hz}, 1 \mathrm{H}, 4^{\prime}-\mathrm{H}\right), 3.28\left(\mathrm{dd}, J=9.6 \mathrm{~Hz}, J=16.0 \mathrm{~Hz}, 1 \mathrm{H}, 4^{\prime}-\mathrm{H}\right), 3.52$ (s, $3 \mathrm{H}$, $\left.\mathrm{OCH}_{3}\right), 3.78\left(\mathrm{~s}, 3 \mathrm{H}, \mathrm{OCH}_{3}\right), 4.23(\mathrm{~d}, J=12.0 \mathrm{~Hz}, 1 \mathrm{H}, 6-\mathrm{H}), 4.50(\mathrm{~d}, J=2.8 \mathrm{~Hz}, 1 \mathrm{H}, 12 \mathrm{a}-\mathrm{H})$, $4.58\left(\mathrm{dd}, 1 \mathrm{H}, J=2.4 \mathrm{~Hz}, J=12.4 \mathrm{~Hz}, \mathrm{CH}_{2} \mathrm{Cl}\right), 4.64\left(\mathrm{~s}, 2 \mathrm{H}, 6 \mathrm{H}, \mathrm{CH}_{2} \mathrm{Cl}\right), 4.81(\mathrm{~d}, J=3.2 \mathrm{~Hz}, 1 \mathrm{H}$, $6 \mathrm{a}-\mathrm{H}), 4.90\left(\mathrm{~s}, 1 \mathrm{H}, 7^{\prime}-\mathrm{H}\right), 5.06\left(\mathrm{~s}, 1 \mathrm{H}, 7^{\prime}-\mathrm{H}\right), 5.15\left(\mathrm{t}, J=8.8 \mathrm{~Hz}, 1 \mathrm{H}, 5^{\prime}-\mathrm{H}\right), 5.23(\mathrm{~d}, J=12.4$ $\left.\mathrm{Hz}, 1 \mathrm{H}, \mathrm{NOCH}_{2}\right), 5.32\left(\mathrm{~d}, J=12.4 \mathrm{~Hz}, 1 \mathrm{H}, \mathrm{NOCH}_{2}\right), 6.40(\mathrm{~s}, 1 \mathrm{H}, 4-\mathrm{H}), 6.45(\mathrm{~d}, J=8.8 \mathrm{~Hz}, 1 \mathrm{H}$, $10-\mathrm{H}), 6.48(\mathrm{~s}, 1 \mathrm{H}, 1-\mathrm{H}), 7.46 \sim 7.58\left(\mathrm{~m}, 8 \mathrm{H}, \mathrm{C}_{6} \mathrm{H}_{4} \mathrm{C}_{6} \mathrm{H}_{4}\right), 7.80$ (d, $\left.J=8.8 \mathrm{~Hz}, 1 \mathrm{H}, 11-\mathrm{H}\right)$. Anal. calcd for $\mathrm{C}_{37} \mathrm{H}_{34} \mathrm{ClNO}_{6}: \mathrm{C}, 71.20 \%, \mathrm{H}, 5.49 \%, \mathrm{~N}, 2.24 \%$. Found: C, $71.31 \%, \mathrm{H}, 5.45 \%, \mathrm{~N}$, $2.32 \%$. IR (KBr) v/cm ${ }^{-1}: 3017,2975,2938,2912,2852,1675,1620,1511,1197,780,731$.

Compound 3f. White solid. ${ }^{1} \mathrm{H}$ NMR $\left(\mathrm{CDCl}_{3}, 400 \mathrm{MHz}\right) \delta$ : $1.75\left(\mathrm{~s}, 3 \mathrm{H}, 8{ }^{\prime}-\mathrm{CH}_{3}\right), 2.91$ (dd, $J=$ $\left.8.0 \mathrm{~Hz}, J=16.0 \mathrm{~Hz}, 1 \mathrm{H}, 4^{\prime}-\mathrm{H}\right), 3.27\left(\mathrm{dd}, J=9.6 \mathrm{~Hz}, J=16.0 \mathrm{~Hz}, 1 \mathrm{H}, 4^{\prime}-\mathrm{H}\right), 3.52$ (s, 3H, $\left.\mathrm{OCH}_{3}\right), 3.78\left(\mathrm{~s}, 3 \mathrm{H}, \mathrm{OCH}_{3}\right), 4.22(\mathrm{~d}, J=12.0 \mathrm{~Hz}, 1 \mathrm{H}, 6-\mathrm{H}), 4.49(\mathrm{~d}, J=2.4 \mathrm{~Hz}, 1 \mathrm{H}, 12 \mathrm{a}-\mathrm{H})$, $4.58\left(\mathrm{~m}, 3 \mathrm{H}, 6-\mathrm{H}, \mathrm{C}_{6} \mathrm{H}_{4} \mathrm{CH}_{2} \mathrm{Cl}\right), 4.78(\mathrm{~d}, J=3.6 \mathrm{~Hz}, 1 \mathrm{H}, 6 \mathrm{a}-\mathrm{H}), 4.90\left(\mathrm{~s}, 1 \mathrm{H}, 7^{\prime}-\mathrm{H}\right), 5.06(\mathrm{~s}, 1 \mathrm{H}$, $\left.7^{\prime}-\mathrm{H}\right), 5.15\left(\mathrm{t}, J=8.8 \mathrm{~Hz}, 1 \mathrm{H}, 5^{\prime}-\mathrm{H}\right), 5.23\left(\mathrm{~d}, J=12.0 \mathrm{~Hz}, 1 \mathrm{H}, \mathrm{NOCH}_{2}\right), 5.32(\mathrm{~d}, J=12.0 \mathrm{~Hz}$, $\left.1 \mathrm{H}, \mathrm{NOCH}_{2}\right), 6.40(\mathrm{~s}, 1 \mathrm{H}, 4-\mathrm{H}), 6.45(\mathrm{~s}, 1 \mathrm{H}, 1-\mathrm{H}), 6.45(\mathrm{~d}, J=8.4 \mathrm{~Hz}, 1 \mathrm{H}, 10-\mathrm{H}), 7.39$ (d, $J=$ $\left.8.0 \mathrm{~Hz}, 2 \mathrm{H}, \mathrm{C}_{6} \mathrm{H}_{4} 2-\mathrm{H}, \mathrm{C}_{6} \mathrm{H}_{4} 6-\mathrm{H}\right), 7.48\left(\mathrm{~d}, J=8.0 \mathrm{~Hz}, 2 \mathrm{H}, \mathrm{C}_{6} \mathrm{H}_{4} 3-\mathrm{H}, \mathrm{C}_{6} \mathrm{H}_{4} 5-\mathrm{H}\right), 7.79$ (d, $J=$ $8.4 \mathrm{~Hz}, 1 \mathrm{H}, 11-\mathrm{H})$. Anal. calcd for $\mathrm{C}_{31} \mathrm{H}_{30} \mathrm{ClNO}_{6}: \mathrm{C}, 67.94 \%, \mathrm{H}, 5.52 \%, \mathrm{~N}, 2.56 \%$. Found: $\mathrm{C}$, $67.90 \%, \mathrm{H}, 5.63 \%, \mathrm{~N}, 2.59 \%$. IR (KBr) $v / \mathrm{cm}^{-1}: 3062,2965,2933,2878,2855,1777,1619$, $1513,1199,776,738$.

Compound 3g. White solid. ${ }^{1} \mathrm{H}$ NMR $\left(\mathrm{CDCl}_{3}, 400 \mathrm{MHz}\right) \delta: 1.75\left(\mathrm{~s}, 3 \mathrm{H}, 8\right.$ ' $\left.-\mathrm{CH}_{3}\right), 2.25(\mathrm{~s}, 6 \mathrm{H}$, $\left.2 \times \mathrm{CH}_{3}\right), 2.41\left(\mathrm{~s}, 3 \mathrm{H}, \mathrm{CH}_{3}\right), 2.91\left(\mathrm{dd}, J=8.4 \mathrm{~Hz}, J=16.0 \mathrm{~Hz}, 1 \mathrm{H}, 4^{\prime}-\mathrm{H}\right), 3.27(\mathrm{dd}, J=10.0 \mathrm{~Hz}$, $J=16.0 \mathrm{~Hz}, 1 \mathrm{H}, 4$ '-H), $3.55\left(\mathrm{~s}, 3 \mathrm{H}, \mathrm{OCH}_{3}\right), 3.78\left(\mathrm{~s}, 3 \mathrm{H}, \mathrm{OCH}_{3}\right), 4.20(\mathrm{~d}, J=12.0 \mathrm{~Hz}, 1 \mathrm{H}, 6-\mathrm{H})$, $4.48(\mathrm{t}, J=2.4 \mathrm{~Hz}, 1 \mathrm{H}, 12 \mathrm{a}-\mathrm{H}), 4.56(\mathrm{dd}, J=2.0 \mathrm{~Hz}, J=12.0 \mathrm{~Hz}, 1 \mathrm{H}, 6-\mathrm{H}), 4.78(\mathrm{~m}, 3 \mathrm{H}, 6 \mathrm{a}-\mathrm{H}$, $\left.\mathrm{CH}_{2} \mathrm{Cl}\right), 4.90\left(\mathrm{~s}, 1 \mathrm{H}, 7^{\prime}-\mathrm{H}\right), 5.05\left(\mathrm{~s}, 1 \mathrm{H}, 7^{\prime}-\mathrm{H}\right), 5.14\left(\mathrm{t}, J=8.8 \mathrm{~Hz}, 1 \mathrm{H}, 5^{\prime}-\mathrm{H}\right), 5.33(\mathrm{~d}, J=11.6$ $\left.\mathrm{Hz}, 1 \mathrm{H}, \mathrm{NOCH}_{2}\right), 5.40\left(\mathrm{~d}, J=11.6 \mathrm{~Hz}, 1 \mathrm{H}, \mathrm{NOCH}_{2}\right), 6.39$ (s, 1H, 4-H), $6.42(\mathrm{~s}, 1 \mathrm{H}, 1-\mathrm{H}), 6.45$ $(\mathrm{d}, J=8.8 \mathrm{~Hz}, 1 \mathrm{H}, 10-\mathrm{H}), 7.18\left(\mathrm{~s}, 3 \mathrm{H}, \mathrm{C}_{6} \mathrm{H}_{4} 3-\mathrm{H}\right), 7.25\left(\mathrm{~d}, J=10.8 \mathrm{~Hz}, 1 \mathrm{H}, \mathrm{C}_{6} \mathrm{H}_{4} 6-\mathrm{H}\right), 7.75(\mathrm{~d}$, $J=8.8 \mathrm{~Hz}, 1 \mathrm{H}, 11-\mathrm{H})$. Anal. calcd for $\mathrm{C}_{33} \mathrm{H}_{34} \mathrm{ClNO}_{6}: \mathrm{C}, 68.80 \%, \mathrm{H}, 5.95 \%, \mathrm{~N}, 2.43 \%$. Found: C, 68.63\%, H, 6.03\%, N, 2.40\%. IR (KBr) v/cm ${ }^{-1}$ : 3082, 2974, 2966, 2937, 2835, 1619, 1507, $1184,964,743$.

Compound 4a. $\mathrm{n}(2): \mathrm{n}\left(\mathrm{RCl}_{2}\right)=2: 1$, white solid. ${ }^{1} \mathrm{H} \mathrm{NMR}\left(\mathrm{CDCl}_{3}, 400 \mathrm{MHz}\right) \delta: 1.75(\mathrm{~s}, 6 \mathrm{H}$, $\left.2 \times 8^{\prime}-\mathrm{CH}_{3}\right), 2.91\left(\mathrm{dd}, J=8.0 \mathrm{~Hz}, J=16.0 \mathrm{~Hz}, 2 \mathrm{H}, 2 \times 4^{\prime}-\mathrm{H}\right), 3.27(\mathrm{dd}, J=9.6 \mathrm{~Hz}, J=16.0 \mathrm{~Hz}$, $2 \mathrm{H}, 2 \times 4$ '- $-\mathrm{H}), 3.57\left(\mathrm{~s}, 6 \mathrm{H}, 2 \times \mathrm{OCH}_{3}\right), 3.78\left(\mathrm{~s}, 6 \mathrm{H}, 2 \times \mathrm{OCH}_{3}\right), 4.22(\mathrm{~d}, J=12.0 \mathrm{~Hz}, 2 \mathrm{H}, 2 \times 6-\mathrm{H})$, $4.49(\mathrm{~d}, J=2.4 \mathrm{~Hz}, 2 \mathrm{H}, 2 \times 12 \mathrm{a}-\mathrm{H}), 4.57$ (dd, $J=2.4 \mathrm{~Hz}, J=12.0 \mathrm{~Hz}, 2 \mathrm{H}, 2 \times 6-\mathrm{H}), 4.78(\mathrm{~d}, J=$ $3.2 \mathrm{~Hz}, 2 \mathrm{H}, 2 \times 6 \mathrm{a}-\mathrm{H}), 4.90\left(\mathrm{~s}, 2 \mathrm{H}, 2 \times 7^{\prime}-\mathrm{H}\right), 5.05\left(\mathrm{~s}, 2 \mathrm{H}, 2 \times 7^{\prime}-\mathrm{H}\right), 5.15\left(\mathrm{t}, J=8.8 \mathrm{~Hz}, 2 \mathrm{H}, 2 \times 5^{\prime}-\right.$ $\mathrm{H}), 5.25\left(\mathrm{~d}, J=12.0 \mathrm{~Hz}, 2 \mathrm{H}, 2 \times \mathrm{OCH}_{2}-\mathrm{H}\right), 5.30\left(\mathrm{~d}, J=12.0 \mathrm{~Hz}, 1 \mathrm{H}, 2 \times \mathrm{OCH}_{2}-\mathrm{H}\right), 6.40(\mathrm{~s}, 2 \mathrm{H}$, $2 \times 4-\mathrm{H}), 6.45(\mathrm{~d}, J=8.4 \mathrm{~Hz}, 2 \mathrm{H}, 2 \times 10-\mathrm{H}), 6.50(\mathrm{~s}, 2 \mathrm{H}, 2 \times 1-\mathrm{H}), 7.45\left(\mathrm{~s}, 4 \mathrm{H}, 2 \times \mathrm{C}_{6} \mathrm{H}_{4}-\mathrm{H}\right), 7.78(\mathrm{~d}$, $J=8.4 \mathrm{~Hz}, 2 \mathrm{H}, 2 \times 11-\mathrm{H})$. Anal. calcd for $\mathrm{C}_{54} \mathrm{H}_{52} \mathrm{~N}_{2} \mathrm{O}_{12}: \mathrm{C}, 70.42 \%, \mathrm{H}, 5.69 \%, \mathrm{~N}, 3.04 \%$. Found: C, $70.23 \%, \mathrm{H}, 5.64 \%$, N, 2.99\%. IR (KBr) $v / \mathrm{cm}^{-1}: 3061,3017,2929,2983,2870,1675,1620$, $1510,1201,1092,982,740$.

Compound 4b. $\mathrm{n}(2): \mathrm{n}\left(\mathrm{RCl}_{2}\right)=2: 1$, white solid. ${ }^{1} \mathrm{H} \mathrm{NMR}\left(\mathrm{CDCl}_{3}, 400 \mathrm{MHz}\right) \delta: 1.76(\mathrm{~s}, 6 \mathrm{H}$, $\left.2 \times 8^{\prime}-\mathrm{CH}_{3}\right), 2.92\left(\mathrm{dd}, J=8.0 \mathrm{~Hz}, J=16.0 \mathrm{~Hz}, 2 \mathrm{H}, 2 \times 4^{\prime}-\mathrm{H}\right), 3.24(\mathrm{dd}, J=9.6 \mathrm{~Hz}, J=16.0 \mathrm{~Hz}$, $\left.2 \mathrm{H}, 2 \times 4^{\prime}-\mathrm{H}\right), 3.52\left(\mathrm{~s}, 6 \mathrm{H}, 2 \times \mathrm{OCH}_{3}\right), 3.78\left(\mathrm{~s}, 6 \mathrm{H}, 2 \times \mathrm{OCH}_{3}\right), 4.22(\mathrm{~d}, J=12.0 \mathrm{~Hz}, 2 \mathrm{H}, 2 \times 6-\mathrm{H})$, $4.51(\mathrm{~s}, 2 \mathrm{H}, 2 \times 12 \mathrm{a}-\mathrm{H}), 4.58(\mathrm{dd}, 2 \mathrm{H}, J=2.4 \mathrm{~Hz}, J=12.0 \mathrm{~Hz}, 2 \times 6-\mathrm{H}), 4.82(\mathrm{~d}, J=2.8 \mathrm{~Hz}, 2 \mathrm{H}$, $2 \times 6 \mathrm{a}-\mathrm{H}), 4.90\left(\mathrm{~s}, 2 \mathrm{H}, 2 \times 7^{\prime}-\mathrm{H}\right), 5.06\left(\mathrm{~s}, 2 \mathrm{H}, 2 \times 7^{\prime}-\mathrm{H}\right), 5.13\left(\mathrm{t}, J=8.8 \mathrm{~Hz}, 2 \mathrm{H}, 2 \times 5^{\prime}-\mathrm{H}\right), 5.28(\mathrm{~d}$, $\left.J=12.0 \mathrm{~Hz}, 2 \mathrm{H}, \mathrm{OCH}_{2}\right), 5.36\left(\mathrm{~d}, J=12.0 \mathrm{~Hz}, 2 \mathrm{H}, \mathrm{OCH}_{2}\right), 6.40(\mathrm{~s}, 2 \mathrm{H}, 2 \times 4-\mathrm{H}), 6.45(\mathrm{~d}, J=8.4$ $\mathrm{Hz}, 2 \mathrm{H}, 2 \times 10-\mathrm{H}), 6.49(\mathrm{~s}, 2 \mathrm{H}, 2 \times 1-\mathrm{H}), 7.58\left(\mathrm{~m}, 8 \mathrm{H}, 2 \times \mathrm{C}_{6} \mathrm{H}_{4}\right), 7.81(\mathrm{~d}, J=8.4 \mathrm{~Hz}, 2 \mathrm{H}, 11-\mathrm{H})$. 
Anal. calcd for $\mathrm{C}_{60} \mathrm{H}_{56} \mathrm{~N}_{2} \mathrm{O}_{12}: \mathrm{C}, 72.27 \%, \mathrm{H}, 5.66 \%, \mathrm{~N}, 2.81 \%$. Found: C, 73.05\%, H, 5.62\%, N, $2.72 \%$. IR (KBr) $v / \mathrm{cm}^{-1}: 3018,2972,2935,2912,2860,1677,1621,1513,1202,780,741$.

Compound 4c. $\mathrm{n}(2): \mathrm{n}\left(\mathrm{RCl}_{2}\right)=2: 1$, white solid. ${ }^{1} \mathrm{H} \mathrm{NMR}\left(\mathrm{CDCl}_{3}, 400 \mathrm{MHz}\right) \delta: 1.75(\mathrm{~s}, 6 \mathrm{H}$, $2 \times 8$ ' $\left.-\mathrm{CH}_{3}\right), 2.41\left(\mathrm{~s}, 6 \mathrm{H}, \mathrm{CH}_{3}\right), 2.90(\mathrm{dd}, J=8.4 \mathrm{~Hz}, J=16.0 \mathrm{~Hz}, 2 \mathrm{H}, 2 \times 4 '-\mathrm{H}), 3.25$ (dd, $J=9.6$ $\left.\mathrm{Hz}, J=16.0 \mathrm{~Hz}, 2 \mathrm{H}, 2 \times 4^{\prime}-\mathrm{H}\right), 3.58\left(\mathrm{~s}, 6 \mathrm{H}, 2 \times \mathrm{OCH}_{3}\right), 3.78\left(\mathrm{~s}, 6 \mathrm{H}, 2 \times \mathrm{OCH}_{3}\right), 4.22(\mathrm{~d}, J=12.0$ $\mathrm{Hz}, 2 \mathrm{H}, 2 \times 6-\mathrm{H}), 4.46(\mathrm{~s}, 2 \mathrm{H}, 2 \times 12 \mathrm{a}-\mathrm{H}), 4.52(\mathrm{dd}, 2 \mathrm{H}, J=2.0 \mathrm{~Hz}, J=12.0 \mathrm{~Hz}, 2 \times 6-\mathrm{H}), 4.70(\mathrm{~d}$, $J=2.4 \mathrm{~Hz}, 2 \mathrm{H}, 2 \times 6 \mathrm{a}-\mathrm{H}), 4.90\left(\mathrm{~s}, 2 \mathrm{H}, 2 \times 7^{\prime}-\mathrm{H}\right), 5.05\left(\mathrm{~s}, 2 \mathrm{H}, 2 \times 7^{\prime}-\mathrm{H}\right), 5.14(\mathrm{t}, J=8.8 \mathrm{~Hz}, 2 \mathrm{H}$, $2 \times 5$ ' $-\mathrm{H}), 5.23\left(\mathrm{~d}, J=10.8 \mathrm{~Hz}, 2 \mathrm{H}, \mathrm{OCH}_{2}\right), 5.32\left(\mathrm{~d}, J=10.8 \mathrm{~Hz}, 2 \mathrm{H}, \mathrm{OCH}_{2}\right), 6.36(\mathrm{~s}, 2 \mathrm{H}, 2 \times 4-$ $\mathrm{H}), 6.44(\mathrm{~s}, 2 \mathrm{H}, 2 \times 1-\mathrm{H}), 6.38(\mathrm{~d}, J=8.8 \mathrm{~Hz}, 2 \mathrm{H}, 2 \times 10-\mathrm{H}), 7.03\left(\mathrm{~s}, 1 \mathrm{H}, \mathrm{C}_{6} \mathrm{H}_{4} 5\right.$ "- $\left.\mathrm{H}\right), 7.43(\mathrm{~s}, 1 \mathrm{H}$, $\left.\mathrm{C}_{6} \mathrm{H}_{4} 2 "-\mathrm{H}\right), 7.79(\mathrm{~d}, J=8.8 \mathrm{~Hz}, 1 \mathrm{H}, 11-\mathrm{H})$. Anal. calcd for $\mathrm{C}_{56} \mathrm{H}_{56} \mathrm{~N}_{2} \mathrm{O}_{12}: \mathrm{C}, 70.87 \%, \mathrm{H}, 5.95 \%$, $\mathrm{N}, 2.95 \%$. Found: C, $70.72 \%, \mathrm{H}, 5.89 \%, \mathrm{~N}, 2.92 \%$. IR (KBr) v/cm $\mathrm{cm}^{-1}: 3085,2962,2937,2835$, $1676,1513,1415,1260,1324,1173,910,743$.

\section{Bioassay of insecticidal activity}

Test pathogens: Stock colonies of rice green leafhopper N. cincticeps, bean aphid A. fabae, twospot red mite $T$. urticae and oriental armyworm $M$. separata were reared in a conditioned room maintained at $25 \pm 5{ }^{\circ} \mathrm{C}, 65 \pm 5 \%$ relative humidity and 12:12 hour light:dark photoperiod [14]. Stock solutions of each test compound were prepared in acetone at a concentration of $1.000 \mathrm{~g} / \mathrm{L}$, and then diluted to the required test concentrations for use with water containing an appropriate amount of Tween-80.

\section{Insecticidal activity against M. separata}

Potter-spraying was used to measure the insecticidal activity of test compounds against $M$. separata. Fifty third-instar M. separata and five corn fragments were placed in a Petri dish and sprayed with test solutions $(500 \mathrm{mg} / \mathrm{L})$ using a Potter sprayer [15]. After air drying, they were kept in a room for normal cultivation. After 24 hours, the number of live and dead insects was recorded. The test was run three times, and the results were averaged.

\section{Insecticidal and miticidal activities against N. cincticeps, A. fabae and T. urticae}

To measure the activities of test compounds against $N$. cincticeps, A. fabae and T. urticae, a leaf-dipping method was introduced. Rice seedlings (second semester) were dipped in the test solution $(500 \mathrm{mg} / \mathrm{L})$ for 5 seconds, air-dried and then placed in a large test tube. Each test tube contained 20 seedlings. Twenty leafhoppers (third instar lymph) were introduced into the tube, and the mouth of the tube was covered with white cheesecloth. The tube was kept at room temperature, and the number of live and dead insects counted after 24 hours. The test was run three times, and the results were averaged. The mortality was determined by the number of live and dead insects as follows:

$$
M=\frac{\bar{N}_{1}}{N_{0}} \times 100 \%
$$

in which $\mathrm{M}$ is the mortality, $\mathrm{N}_{0}$ is the total number of live insects for the test, and $\bar{N}_{1}$ is the average number of dead insects.

\section{Bioassay of fungicidal activity}

Fungicidal activity of the title compounds against Gibberella zeae (G. zeae), Phytophthora capsici (P. capsici), Pyricularia oryzae (P. oryzae), Botrytis cinerea (B. cinerea), Sclerotinia sclerotiorum ( $S$. sclerotiorum), Rhizoctonia solani (R. solani) and Erysiphe graminis (E. 
graminis) were evaluated using the mycelium growth rate test [16]. The appropriate amount of test compounds was weighed accurately, dissolved in the acetone and then diluted into 500 $\mathrm{mg} / \mathrm{L}$. The culture media, with a known concentration of the test compounds, were obtained by mixing the solution of test compounds in acetone with potato dextrose agar (PDA), on which fungus cakes were placed. The blank test was made using acetone. The culture was grown at $25 \pm 5{ }^{\circ} \mathrm{C}, 65 \pm 5 \%$ relative humidity and $12: 12$ hour light:dark photoperiod for 72 hours. Three replicates were performed. After the mycelia grew completely, the diameter of the mycelia was measured and the inhibition rate calculated according to the formula:

$$
I=\frac{\bar{D}_{1}-\bar{D}_{0}}{\bar{D}_{1}} \times 100 \%
$$

In which $I$ is the inhibition rate, $\bar{D}_{1}$ is the average diameter of mycelia in the blank test, and $\bar{D}_{0}$ is the average diameter of mycelia in the presence of the test compounds.

\section{ACKNOWLEDGEMENTS}

The authors gratefully thank the Natural Science Foundation of Guangdong Province (No. 10451022401005172) for their financial support for this project.

\section{REFERENCES}

1. Brown, C.D.; Hodgkinson, R.A.; Rose, D.A.; Syers, J.K.; Wilcockson, S.J. Pestic. Sci. 1995, 43, 131.

2. Fang, N.; Casida, J.E. J. Org. Chem. 1997, 62, 350.

3. Zhou, Z.Z.; You, W.W. Chin. J. Org. Chem. 2008, 28, 1849.

4. Cabras, P.; Caboni, P.; Cabras, M.; Angioni, A.; Russo, M. J. Agric. Food. Chem. 2002, 50, 2576.

5. Cheng, H.M.; Yamamoto, I.; Casida, J.E. J. Agr. Food. Chem. 1972, 20, 850.

6. Yamamoto, I.; Unai, T.; Ohkawa, H.; Casida, J.E. Pestic. Biochem. Physiol. 1971, 1, 143.

7. Coll, J. J. Agric. Food. Chem. 2005, 53, 3749.

8. Ueno, H.; Miyoshi, H.; Inoue, M.; Niidome, Y.; Iwamura, H. BBA-Bioenergetics 1996, 1276, 195.

9. Ye, J.; Peng, J.M.; Hu, A.X.; Wang, C.; Zou, M; Ou, X.M. Chin. J. Chem. 2011, 6, 886.

10. Pelly, S.C.; Govender, S.; Fernandes, M.A.; Schmalz, H.G.; de Koning, C.B. J. Org. Chem. 2007, 72, 2857.

11. Ye, J.; Wang, C.; Hu, A.X.; Xu, H.H.; Ou, X.M. Chinese J. Struct. Chem. 2010, 29, 297.

12. Kostova, I. Liebigs Ann. Chem. 1988, 3, 195.

13. Yang, S.P.; Yu, X.B.; Huang, J.G.; Xu, H.H. Acta. Cryst. 2003, C57, 0392.

14. Liu, A.P.; Ou, X.M.; Huang, M.Z.; Wang, X.G.; Liu, X.P.; Wang, Y.J.; Chen, C.; Yao, J.R. Pest. Manag. Sci. 2005, 61, 166.

15. Yenesew, A.; Mushibe, E.K.; Induli, M.; Derese, S.; Midiwo, J.O.; Kabaru, J.M.; Heydenreich, M.; Koch, A.; Peter, M.G. Phytochemistry 2005, 66, 653.

16. Clough, J.M.; Godfrey, C.R. The Strobilurin Fungicides: Fungicidal Activity, Chemical and Biological Approaches to Plant Protection, John Wiley and Sons: Surrey, U.K.; 1998. 